Research Article

J Exp Clin Med

2021; 38(4): 577-582

doi: $10.52142 /$ omujecm.38.4.32

\title{
Are neutrophil count and neutrophil/lymphocyte ratio useful as markers of polycystic ovary syndrome in early reproductive age?
}

\author{
Sabri ÇOLAK*iD, Beril GÜRLEK(i)
}

Department of Obstetrics and Gynecology, Faculty of Medicine, Recep Tayyip Erdogan University, Rize, Turkey

\begin{abstract}
\begin{tabular}{ccccc}
\hline Received: $20.04 .2021 \quad \bullet \quad$ Accepted/Published Online: 03.05.2021 $\quad$ Final Version: 30.08 .2021
\end{tabular}
\section{Abstract}

Necrotizing fasciitis (NF) is often fatal, characterized by extensive necrosis of the subcutaneous tissues and fascia. The present study was aimed to validate the Laboratory Risk Indicator for Necrotising Fasciitis (LRINEC) score as a tool to predict/diagnose NF and to differentiate it from other soft tissue infections depending on the score. A Prospective Observational study was conducted in ESICMC PGI MSR, Medical College Hospital, Rajajinagar, Bengaluru, from Jan 2019 to June 2020. Patients $\geq 18$ years of age with severe soft tissue infections were included in the study. Based on the LRINEC score, the patients were categorised as low $(\leq 5)$, moderate (6-7) and high risk $(\geq 8)$ for the prediction of onset or diagnosis of NF. Data analysis was performed using SPSS version 21.0. A total of 55 patients were included in the study. A significant association was observed with age ( $\mathrm{p}=0.042)$, LRINEC score $(\mathrm{p}=0.0001)$, C Reactive Protein (CRP; $\mathrm{p}=0.0001)$, haemoglobin $(\mathrm{p}=0.008)$, serum sodium levels $(\mathrm{p}=0.004)$, serum creatinine $(0.001)$, and amputation $(\mathrm{p}=0.004)$. Amputation was done in 5 cases. Only 1 mortality was observed in LRINEC high risk group with NSSTI. To conclude, LRINEC scoring system showed a better positive predictive value in identifying the onset of NF and risk strategizing of the patients with severe soft tissue infections. This study has aimed to contribute to the literature by investigating the value of inflammatory biomarkers of polycystic ovary syndrome (PCOS) that can be tested via a complete blood count. This retrospectively designed case-control study included 197 women in early reproductive age; who were in the age range of 18-24 years and who were admitted to the gynecology outpatient clinic. A total of 111 PCOS patients; in whom the diagnosis of PCOS was made based on Rotterdam criteria, were included in the study. A control group was formed by including 86 healthy women. All measurements of inflammatory biomarkers were obtained from the complete blood count test results. Of the inflammatory markers; the neutrophil count and the neutrophil/lymphocyte ratio were statistically significantly higher in the PCOS group compared to the control group ( $\mathrm{p}=0.016$ and $\mathrm{p}=0.002$, respectively); however, the measured values of other parameters were similar between two groups. To evaluate whether or not the neutrophil count and neutrophil/lymphocyte ratio could be used as a screening tool to exclude PCOS, we constructed a receiver-operating characteristic curve (ROC). The ROC curve for the neutrophil count was $0.60(\mathrm{p}=0.016)$ and NLR was $0.627(\mathrm{p}=0.002)$. The neutrophil count and NLR were higher in the PCOS cases compared to the age-matched individuals in the control group. This finding confirms the presence of inflammation in PCOS cases of early reproductive age. However, it has been demonstrated that the diagnostic values of these markers are not strong in distinguishing PCOS patients from healthy individuals.
\end{abstract}

Keywords: Inflammatory markers, infertility, lymphocyte, neutrophil, polycystic ovary syndrome, ratio

\section{Introduction}

Polycystic ovary syndrome (PCOS) is a metabolic and ovulatory disorder; which becomes manifest in adolescence (1) and continues throughout the reproductive ages, causing infertility in most patients (2).

The major factor involved in the pathogenesis of both clinical and biochemical features of PCOS is the metabolic disorder caused by increased quantities of adipose tissue as observed in most cases. The emerging evidence points out the fact that metabolic changes such as hyperandrogenism, type 2 diabetes, insulin resistance, and cardiovascular disease occurring during the course of PCOS in the long term may be associated with low-grade chronic inflammatory environment (3-6). Studies have shown that a significant increase occurs in circulating inflammatory marker levels in PCOS and that proinflammation is an important component of $\operatorname{PCOS}(7,8)$. It is known that inflammation affects many complex mechanisms and that detection of an increase in immune cells may indicate inflammation. In clinical terms, studies have shown that an increase in inflammatory biomarker levels in the complete blood count may be an indicator of inflammation $(6,9,10)$. A literature review reveals that; of the hematological biomarkers, the neutrophil-to-lymphocyte ratio (NLR) may have prognostic significance in many cancers and conditions affecting the metabolic and cardiac systems (11-14). Studies examining the significance of hematological inflammatory biomarkers in different clinical phenotypes of PCOS found out that the levels of some biomarkers including the counts of white blood cells, neutrophils, leukocytes, and the mean platelet volume increased in the PCOS group (15-17). However, the results in 
the literature are controversial (18). Therefore; this study has aimed to contribute to the literature by investigating the value of inflammatory biomarkers that can be tested via a complete blood count but having no established diagnostic significance, yet.

\section{Materials and methods}

\subsection{Study design and patient selection}

This retrospectively designed case-control study included 197 women in early reproductive age; who were in the age range of 18-24 years and who were admitted to the gynecology outpatient clinic of Recep Tayyip Erdoğan University School of Medicine's Training and Research Hospital in the period between January 2016 and December 2019.

In accordance with the World Medical Association's 2008-revised version of the 1975-version of the Declaration of Helsinki, the study commenced after obtaining approval from the Ethics Committee of Recep Tayyip Erdoğan University (Approval No:2020/48).

Data; including the medical history, habits, and anthropometric parameters of participants in the PCOS and healthy control groups were obtained from hospital records. Our study excluded participants with the following conditions; including hyperandrogenism-related diseases such as androgen-secreting tumors, congenital adrenal hyperplasia, Cushing's syndrome, hyperprolactinemia, and thyroid diseases; liver and kidney dysfunction, cardiovascular diseases, diabetes mellitus, chronic inflammatory disease, malignancy, pregnancy, acquired immune deficiency syndrome, and nicotine and alcohol consumption. Participants using drugs with potential effects on the inflammatory state such as oral contraceptives, steroid hormones, insulinsensitizing drugs, antibiotics, or anti-inflammatory drugs were excluded, too.

A total of 111 PCOS patients; in whom the diagnosis of PCOS was made based on Rotterdam criteria, were included in the study. According to the Rotterdam criteria, making a diagnosis of PCOS requires the presence of at least two of the following three symptoms: oligomenorrhea/polymenorrhea; [2] biochemical hyperandrogenis (Ferriman-Gallwey scores (FCS) of $\geq 8$ ) (19) or the presence of clinical hyperandrogenism; [3] detection of polycystic ovaries in an ultrasonographic examination (20).

Blood pressure values, waist and hip circumferences, and the height and weight of participants were retrieved from hospital records. Body mass index (BMI) was calculated using the following formula: BMI=weight $(\mathrm{kg}) /$ height $(\mathrm{m})^{2}$. Complete blood counts and hormonal and biochemical tests were performed using the serum samples taken in the early follicular phase of the menstrual cycle. The results were retrieved from hospital records. All measurements including the counts of total white blood cells, neutrophils, monocytes, platelets, and lymphocytes, and all calculated ratios were obtained from the complete blood count test results.

The homeostatic model assessment-insulin resistance (HOMA-IR) values were calculated using the following formula: HOMA-IR = The fasting blood glucose level $\mathrm{x}$ the fasting insulin level / 22.5 (21).

\subsection{Statistical analysis}

Data were analyzed using the Statistical Package for the Social Sciences software version 23.0 (SPSS IBM, Armonk, NY, USA). Descriptive data were expressed as mean \pm standard deviation. The chi-square test was used in the analysis of categorical variables. The normal distribution of the variables was tested by Kolmogorov-Smirnov test. The nonparametric distribution of the variables was tested by the Mann-Whitney $U$ test. The optimal cut-off points for inflammatory parameters in distinguishing the PCOS patients were further evaluated by receiver operating characteristic curve (ROC) analyses. A probability level of $p<.005$ was considered statistically significant.

\section{Results}

Of the participants included in the study, 111 patients were included in the PCOS group and 86 participants were included in the control group. The anthropometric properties and hormonal and metabolic profiles of the PCOS and control groups are listed in Table 1. The age distribution was statistically found to be similar between the PCOS and control groups $(p=0.104)$. The waist circumference and BMI were statistically significantly higher in the PCOS group compared to the control group ( $p=0.001, p=0.001$, respectively); however, the hip circumference was similar between the two groups $(p=0.082)$. Compared to healthy women; FGS values, as an indicator of clinical hirsutism, were higher in PCOS patients $(\mathrm{p}<0.001)$.

Glucose and insulin values and HOMA-IR scores were not significantly different between the PCOS and control groups. As for the lipid profiles; triglyceride levels were statistically significantly higher in the PCOS group $(p=0.007)$ but the levels of high-density lipoprotein cholesterol (HDLC), low-density lipoprotein cholesterol (LDL-C), and total cholesterol (TC) were not different between the groups. Women with PCOS had significantly higher levels of luteinizing hormone ( $\mathrm{LH})$, total testosterone $(\mathrm{tT})$, and free testosterone (fT) compared to the control group ( $p=0.031$, $\mathrm{p}=0.004$, and $\mathrm{p}=0.001$; respectively). The levels of 17 hydroxyprogesterone (17-OH PG), dehydroepiandrosterone sulfate (DHEA-S), prolactin, and thyroid-stimulating hormone (TSH) were not significantly different between the groups.

Of the inflammatory markers; the neutrophil count and the NLR were statistically significantly higher in the PCOS group compared to the control group $(\mathrm{p}=0.016$ and $\mathrm{p}=0.002$, respectively); however, the measured values of other parameters were similar between the two groups. To evaluate 
whether or not the neutrophil count and NLR could be used as a screening tool to exclude PCOS, we constructed a receiveroperating characteristic (ROC) curve (Fig. 1). Using a cut-off value of 4.955 , the neutrophil count in the serum had a likelihood of excluding PCOS with a sensitivity of $0.39 \%$ and a specificity of $0.81 \%$. Using a cut-off value of 1.755 , NLR in the serum had a likelihood of excluding PCOS with a sensitivity of $0.64 \%$ and a specificity of $0.62 \%$ (Fig. 1 ). The area under the ROC curve for the neutrophil count was 0.60 (95\% CI: 0.512-0.680, $\mathrm{p}=0.016)$. The area under the curve for NLR was 0.627 (95\% CI: $0.549-0.706, p=0.002)$ (Fig.1).

Table 1. The anthropometric and biochemical findings in the polycystic ovary syndrome and control groups

\begin{tabular}{|c|c|c|c|}
\hline Parameters & $\operatorname{PCO}(n=111)$ & Control $(n=86)$ & p-value \\
\hline Age (year) & $21.13 \pm 2.60$ & $21.72 \pm 2.43$ & 0.104 \\
\hline $\mathrm{WC}(\mathrm{cm})$ & $77.72 \pm 10.22$ & $73.36 \pm 9.62$ & $0.001^{*}$ \\
\hline $\mathrm{HC}(\mathrm{cm})$ & $101.30 \pm 9.27$ & $98.85 \pm 9.22$ & 0.082 \\
\hline BMI $\left(\mathrm{kg} / \mathrm{m}^{2}\right)$ & $23.78 \pm 4.88$ & $21.69 \pm 3.49$ & $0.001^{*}$ \\
\hline FGS & $7.77 \pm 3.5$ & $1.85 \pm 2.69$ & $<0.001^{*}$ \\
\hline Glucose (mg/dl) & $90.14 \pm 7.01$ & $91.73 \pm 10.53$ & 0.454 \\
\hline Insulin (mIU/ml) & $10.62 \pm 7.46$ & $9.39 \pm 5.26$ & 0.350 \\
\hline HOMA-IR & $2.36 \pm 1.84$ & $2.04 \pm 1.27$ & 0.238 \\
\hline Triglyceride (mg/dl) & $82.50 \pm 36.41$ & $68.44 \pm 31.19$ & $0.007^{*}$ \\
\hline $\mathrm{TC}(\mathrm{mg} / \mathrm{dl})$ & $181.04 \pm 28.85$ & $199.15 \pm 184.07$ & 0.153 \\
\hline HDL-C (mg/dl) & $60.47 \pm 13.32$ & $62.72 \pm 13.67$ & 0.339 \\
\hline LDL-C (mg/dl) & $102.47 \pm 28.79$ & $99.72 \pm 31.72$ & 0.149 \\
\hline LH (mIU/ml) & $5.7 \pm 5.22$ & $5.7 \pm 5.22$ & $0.031^{*}$ \\
\hline $\mathrm{tT}(\mathrm{ng} / \mathrm{ml})$ & $44.67 \pm 16.43$ & $38.78 \pm 11.49$ & $0.004^{*}$ \\
\hline $\mathrm{fT}(\mathrm{ng} / \mathrm{ml})$ & $2.50 \pm 1.09$ & $2.06 \pm 1.02$ & $0.001^{*}$ \\
\hline 17 OH P (ng/ml) & $1.16 \pm 0.46$ & $2.27 \pm 10.89$ & 0.264 \\
\hline DHEA-S (mcg/dl) & $275.01 \pm 110.02$ & $257.06 \pm 134.84$ & 0.370 \\
\hline TSH (microIU/ml) & $1.68 \pm 0.81$ & $1.73 \pm 0.82$ & 0.772 \\
\hline Prolactin (ng/ml) & $20.40 \pm 14.69$ & $22.74 \pm 35.81$ & 0.375 \\
\hline
\end{tabular}

BMI, body mass index; DHEAS, dehydroepiandrosterone sulfate; FGS, Ferriman-Gallwey Score; fT, free testosterone; HC, hip circumference; HDL-C, high-density lipoprotein cholesterol; HOMA-IR, homeostasis model assessment of insulin resistance; LDL-C, low-density lipoprotein cholesterol; LH, luteinizing hormone; TC, total cholesterol; TSH, thyroid-stimulating hormone; tT, total testosterone; $17 \mathrm{OH}$ P, $17-$ hydroxyprogesterone; $\mathrm{WC}$, waist circumference A p-value of $<0.05$ was accepted as statistically significant

\section{Discussion}

In our study, we found that; of the inflammatory biomarkers in the complete blood count, the neutrophil count and NLR were statistically higher in PCOS patients compared to the control group. In addition, we found that the neutrophil count and NLR values are poorly sensitive biomarkers to be used to distinguish PCOS patients from individuals in the control group. PCOS is a complex disease accompanied by metabolic and ovulatory dysfunction, obesity, and inflammation (7).
Levels of circulating inflammatory markers have been investigated in PCOS. Studies in the literature have reported that levels of proinflammatory cytokines such as IL-1 $\alpha$, IL$1 \beta$, TNF-alpha, IL-6, IL-18, and CRP increase in the systemic circulation. This finding has been recognized as evidence of chronic inflammation in PCOS (22-24). However, the search for an inexpensive and easily accessible new marker to be used in making a diagnosis of PCOS is still a matter of interest for researchers.

Table 2. The levels of the measured blood parameters in the polycystic ovary syndrome and control groups

\begin{tabular}{|l|c|c|c|}
\hline \multicolumn{1}{|c|}{ Parameters } & PCO $(\mathbf{n = 1 1 1})$ & Control (n=86) & p \\
\hline White blood cell count $\left(10^{3} / \mathrm{uL}\right)$ & $7.88 \pm 2.54$ & $7.31 \pm 2.19$ & 0.094 \\
\hline Monocyte count $\left(10^{3} / \mathrm{uL}\right)$ & $0.64 \pm 0.79$ & $0.66 \pm 0.96$ & 0.401 \\
\hline Lymphocyte count $\left(10^{3} / \mathrm{uL}\right)$ & $2.24 \pm 0.79$ & $2.39 \pm 0.79$ & 0.362 \\
\hline Neutrophil count $\left(10^{3} / \mathrm{uL}\right)$ & $4.89 \pm 2.31$ & $4.13 \pm 1.67$ & $0.016^{*}$ \\
\hline Platelet count $\left(\mathrm{x} 10^{3} / \mathrm{mm}^{3}\right)$ & $258.41 \pm 73.14$ & $257.10 \pm 81.24$ & 0.722 \\
\hline Red cell distribution width (fl) & $41.70 \pm 3.68$ & $42.70 \pm 6.56$ & 0.697 \\
\hline Mean platelet volume (fL) & $9.64 \pm 1.62$ & $9.41 \pm 1.54$ & 0.210 \\
\hline Neutrophil/lymphocyte ratio & $2.62 \pm 2.53$ & $1.84 \pm 0.83$ & $0.002^{*}$ \\
\hline Platelet/lymphocyte ratio & $130.13 \pm 63.34$ & $112.94 \pm 36.17$ & 0.149 \\
\hline Monocyte $/$ lymphocyte ratio & $0.31 \pm 0.31$ & $0.28 \pm 0.38$ & 0.049 \\
\hline Mean platelet volume/ lymphocyte ratio $\left(\mathrm{fl} / 10^{3} / \mathrm{uL}\right)$ & $5.03 \pm 2.76$ & $4.31 \pm 1.51$ & 0.103 \\
\hline p-Valle & & & \\
\hline
\end{tabular}

p-value of $<0.05$ was accepted as statistically significant. 


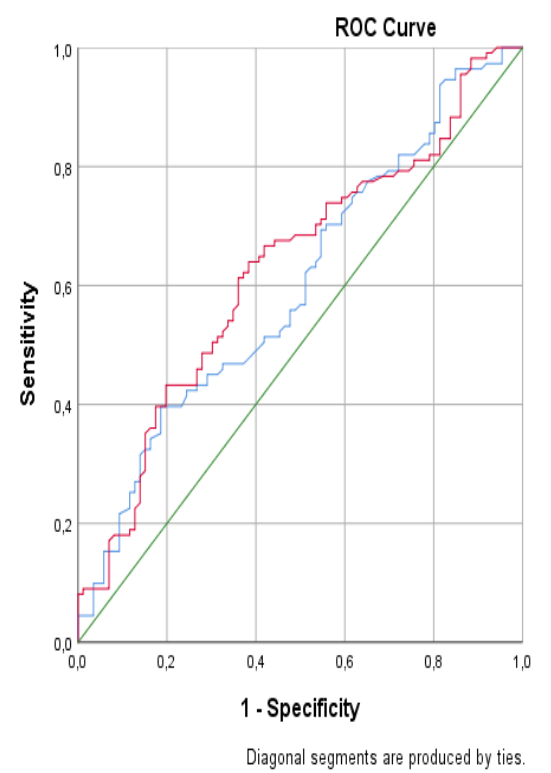

Fig. 1. The receiver-operating characteristic (ROC) curve to evaluate whether the neutrophil count and NLR can be used as diagnostic markers to exclude PCOS

Inflammatory marker levels derived from complete blood count tests and the ratios of such marker levels to each other are evaluated in most medical conditions as inexpensive and easily accessible methods to be used both in the diagnosis and the follow-up of the disease course. Being a parameter obtained from complete blood count tests as the ratio of the neutrophil count to the lymphocyte count; in the literature, NLR is correlated with the activation of diseases accompanied by chronic systemic inflammation including systemic hypertension, atherosclerosis, chronic obstructive pulmonary disease, and systemic lupus erythematosus (2528). There are studies, too, emphasizing that the complete blood count parameters can be used in the diagnosis of PCOS, in the pathophysiology of which low-grade chronic inflammation is thought to have a role similar to the abovementioned diseases $(29,30)$. Studies have shown that; of the complete blood count parameters, especially both the WBC count and the neutrophil count occur at statistically higher levels in the PCOS group compared to the control group $(10,31,32)$. In their study, Pergialiotis et al. found out that both the platelet-to-lymphocyte ratio (PLR) and NLR were correlated with some hormonal and metabolic indicators in PCOS cases (16). In another study, Yllmaz et al. found that neutrophil and basophil counts, NLR, and mean platelet volumes as the inflammatory biomolecules obtained from complete blood count tests were higher in the PCOS group compared to the control group (33). In another study by Rudnicka et al., PCOS cases were compared with healthy individuals in similar groups in terms of age and BMI. That study reported high WBC counts and CRP levels indicating the presence of low-grade inflammation (30). In our study; similar to the information in the literature, the neutrophil count and NLR were higher in PCOS cases of early reproductive age compared to the control group. However; levels of other parameters, including WBC counts, were not significantly different in the PCOS group. A review of the literature reveals studies suggesting that NLR can be an independent prognostic factor in many conditions under the influence of chronic inflammation and that NLR is an important marker to determine the severity of inflammation $(25,34)$. Different from the previous studies in the literature, our study may have provided evidence that only a high neutrophil count and a high NLR may play a more important role as biomarkers showing the impact of inflammation in PCOS compared to all other parameters of a complete blood count test. It can be thought that; compared to the other parameters in a complete blood count test, the neutrophil count and NLR may perform better as diagnostic criteria indicating the degree of inflammation severity and chronic effects of inflammation in patients with PCOS. In addition, it was determined in our study that both the neutrophil count and NLR have poor diagnostic values in differentiating PCOS cases from healthy individuals. However, this finding suggests that the diagnostic precision of these markers, which are accepted as inflammation indicators, is debatable. Inflammation in PCOS can be manifest at different levels of severity depending on the phenotype. We think that the diagnostic value of the neutrophil count and NLR may have been affected by the heterogeneity of the phenotypic distribution of the included cases in our study.

Our study had some limitations. The major limitation is the retrospective inclusion of PCOS patients in our study. The retrospective inclusion of the patients might cause failures in excluding some diseases with the potential to invoke inflammation. Another limitation is the small sample size resulting in the analysis of the results obtained from a small number of patients. In addition, inflammatory markers were examined without phenotypic grouping of PCOS cases. This prevented us to understand the effects of different phenotypes on the neutrophil count and NLR. Finally; because the PCOS cases included in our study were not grouped according to whether they had obesity or not, it was not possible to evaluate the effect of BMI on inflammatory parameters.

The neutrophil count and NLR were higher in the PCOS cases compared to the age-matched individuals in the control group. This finding confirms the presence of inflammation in PCOS cases of early reproductive age. However, it has been demonstrated that the diagnostic values of these markers are not strong in distinguishing PCOS patients from healthy individuals.

\section{Conflict of interest}

The authors declare that they have no conflict of interest.

\section{Acknowledgments}

None to declare. 


\section{References}

1. Besenek M, Gurlek B. Hyperandrogenism in polycystic ovary syndrome affects psychological well-being of adolescents. J Obstet Gynaecol Res 2021;47(1):137-146 (doi: 10.1111/jog.14444). Epub 2020 Aug 23.

2. Azziz R, Woods KS, Reyna R, Key TJ, Knochenhauer ES, Yildiz BO. The prevalence and features of the polycystic ovary syndrome in an unselected population. J Clin Endocrinol Metab. 2004;89(6):2745-9 (doi:10.1210/jc.2003-032046).

3. González F, Sia CL, Shepard MK, Rote NS, Minium J. Inflammation in response to glucose ingestion is independent of excess abdominal adiposity in normal-weight women with polycystic ovary syndrome. J Clin Endocrinol Metab. 2012;97(11):4071-9 (doi:10.1210/jc.2012-2131).

4. Spritzer PM, Lecke SB, Satler F, Morsch DM. Adipose tissue dysfunction, adipokines, and low-grade chronic inflammation in polycystic ovary syndrome. Reproduction 2015;149(5):R219-27 (doi:10.1530/rep-14-0435).

5. Shorakae S, Teede H, de Courten B, Lambert G, Boyle J, Moran LJ. The emerging role of chronic low-grade inflammation in the pathophysiology of polycystic ovary syndrome. Semin Reprod Med. 2015;33(4):257-69 (doi:10.1055/s-0035-1556568).

6. Orio F Jr, Palomba S, Cascella T, Di Biase S, Manguso F, Tauchmanovà L, et al. The increase of leukocytes as a new putative marker of low-grade chronic inflammation and early cardiovascular risk in polycystic ovary syndrome. J Clin Endocrinol Metab. 2005;90(1):2-5 (doi:10.1210/jc.2004-0628).

7. Duleba AJ, Dokras A. Is PCOS an inflammatory process? Fertil Steril. 2012;97(1):7-12 (doi:10.1016/j.fertnstert.2011.11.023).

8. $\mathrm{Hu}$ W, Qiao J, Yang Y, Wang L, Li R. Elevated C-reactive protein and monocyte chemoattractant protein-1 in patients with polycystic ovary syndrome. Eur J Obstet Gynecol Reprod Biol. 2011;157(1):53-6 (doi:10.1016/j.ejogrb.2011.03.015).

9. Kebapcilar L, Taner CE, Kebapcilar AG, Sari I. High mean platelet volume, low-grade systemic coagulation and fibrinolytic activation are associated with androgen and insulin levels in polycystic ovary syndrome. Arch Gynecol Obstet 2009;280(2):187-93 (doi:10.1007/s00404-008-0884-0).

10. Herlihy AC, Kelly RE, Hogan JL, O'Connor N, Farah N, Turner MJ. Polycystic ovary syndrome and the peripheral blood white cell count. J Obstet Gynaecol 2011;31(3):242-4 (doi:10.3109/01443615.2011.553693).

11. Delcea C, Buzea CA, Dan GA. The neutrophil to lymphocyte ratio in heart failure: a comprehensive review. Rom J Intern Med 2019;57(4):296-314 (doi:10.2478/rjim-2019-0018).

12. Monteiro Júnior JGM, de Oliveira Cipriano Torres D, Filho DCS. Hematological parameters as prognostic biomarkers in patients with cardiovascular diseases. Curr Cardiol Rev 2019;15(4):274-82

(doi:10.2174/1573403x15666190225123544).

13. DiGangi C. Neutrophil-lymphocyte ratio: Predicting cardiovascular and renal complications in patients with diabetes. J Am Assoc Nurse Pract 2016;28(8):410-4 (doi:10.1002/23276924.12366).

14. L Liu R, Zheng S, Yuan Q, Zhu P, Li B, Lin Q, et al. The prognostic significance of combined pretreatment fibrinogen and neutrophil-lymphocyte ratio in various cancers: A systematic review and meta-analysis. Dis Markers 2020;2020:4565379 (doi:10.1155/2020/4565379).

15. Can M, Duran C, Guney I, Elmas H, Ayhan M, Erdem SS. The relationship between glomerular filtration rate, and metabolic and inflammatory parameters in obese and non-obese patients with polycystic ovary syndrome. Clin Investig Arterioscler 2020;32(6):256-62 (doi:10.1016/j.arteri.2020.04.003).

16. Pergialiotis V, Trakakis E, Parthenis C, Hatziagelaki E, Chrelias $\mathrm{C}$, Thomakos $\mathrm{N}$, et al Correlation of platelet to lymphocyte and neutrophil to lymphocyte ratio with hormonal and metabolic parameters in women with PCOS. Horm Mol Biol Clin Investig. 2018;34(3) (doi:10.1515/hmbci-2017-0073).

17. Agacayak E, Tunc SY, Sak S, Basaranoglu S, Yüksel H, Turgut A, et al. Levels of neopterin and other inflammatory markers in obese and non-obese patients with polycystic ovary syndrome. Med Sci Monit 2015;21:2446-55 (doi:10.12659/msm.894368).

18. Aydın GA, Turan Özsoy HG, Ankaralı H, Özgen G, Neşelioğlu $\mathrm{S}$. The association of dynamic thiol-disulfide homeostasis and inflammatory markers in patients with polycystic ovary syndrome. Taiwan J Obstet Gynecol 2020; 59(1):79-84 (doi: 10.1016/j.tjog.2019.11.012)

19. Escobar-Morreale HF. Polycystic ovary syndrome: definition, aetiology, diagnosis and treatment. Nat Rev Endocrinol 2018 May;14(5):270-84.

20. Rotterdam ESHRE/ASRM-Sponsored PCOS Consensus Workshop Group. Revised 2003 consensus on diagnostic criteria and long-term health risks related to polycystic ovary syndrome (PCOS). Hum Reprod 2004;19:41-7 (doi: 10.1093/humrep/deh098).

21. Albareda M, Rodríguez-Espinosa J, Murugo M, de Leiva A, Corcoy R. Assessment of insulin sensitivity and $\beta$-cell function from measurements in the fasting state and during an oral glucose tolerance test. Diabetologia 2000;43:1507-11 (doi: $10.1007 / \mathrm{s} 001250051561)$.

22. Zafari Zangeneh F, Naghizadeh MM, Masoumi M. Polycystic ovary syndrome and circulating inflammatory markers. Int $\mathrm{J}$ Reprod Biomed 2017 ;15(6):375-82.

23. Boulman N, Levy Y, Leiba R, Shachar S, Linn R, Zinder O, et al. Increased C-reactive protein levels in the polycystic ovary syndrome: a marker of cardiovascular disease. J Clin Endocrinol Metab 2004;89(5):2160-5 (doi:10.1210/jc.2003-031096).

24. Kelly CC, Lyall H, Petrie JR, Gould GW, Connell JM, Sattar N. Low grade chronic inflammation in women with polycystic ovarian syndrome. J Clin Endocrinol Metab 2001;86(6):2453-5 (doi:10.1210/jcem.86.6.7580).

25. Paliogiannis $P$, Fois AG, Sotgia S, Mangoni AA, Zinellu E, Pirina $P$, et al. Neutrophil to lymphocyte ratio and clinical outcomes in COPD: recent evidence and future perspectives. Eur Respir Rev 2018;27(147) (doi:10.1183/16000617.0113-2017).

26. Mertoglu C, Gunay M. Neutrophil-lymphocyte ratio and plateletlymphocyte ratio as useful predictive markers of prediabetes and diabetes mellitus. Diabetes Metab Syndr 2017;11 Suppl 1:S127s31 (doi:10.1016/j.dsx.2016.12.021).

27. Adamstein NH, MacFadyen JG, Rose LM, Glynn RJ, Dey AK, Libby $\mathrm{P}$, et al. The neutrophil-lymphocyte ratio and incident atherosclerotic events: analyses from five contemporary randomized trials. Eur Heart J 2021 (doi:10.1093/eurheartj/ehaa1034).

28. Q Qin B, Ma N, Tang Q, Wei T, Yang M, Fu H, et al. Neutrophil to lymphocyte ratio (NLR) and platelet to lymphocyte ratio (PLR) were useful markers in assessment of inflammatory response and disease activity in SLE patients. Mod Rheumatol 2016;26(3):372-6 (doi:10.3109/14397595.2015.1091136).

29. Çakıroğlu Y, Vural F, Vural B. The inflammatory markers in polycystic ovary syndrome: association with obesity and IVF outcomes. J Endocrinol Invest 2016;39(8):899-907 


\section{Colak and Gürlek / J Exp Clin Med}

(doi:10.1007/s40618-016-0446-4).

30. Rudnicka E, Kunicki M, Suchta K, Machura P, Grymowicz M, Smolarczyk R. Inflammatory markers in women with polycystic ovary syndrome. Biomed Res Int 2020;2020:4092470 (doi:10.1155/2020/4092470).

31. Phelan N, O'Connor A, Kyaw Tun T, Correia N, Boran G, Roche $\mathrm{HM}$, et al. Leucocytosis in women with polycystic ovary syndrome (PCOS) is incompletely explained by obesity and insulin resistance. Clin Endocrinol (Oxf) 2013;78(1):107-13 (doi:10.1111/j.1365-2265.2012.04454.x).

32. P Papalou O, Livadas S, Karachalios A, Tolia N, Kokkoris P, Tripolitakis K, et al. White blood cells levels and PCOS: direct and indirect relationship with obesity and insulin resistance, but not with hyperandogenemia. Hormones(Athens) 2015;14(1):91100 (doi:10.14310/horm.2002.1563).

33. Yilmaz MA, Duran C, Basaran M. The mean platelet volume and neutrophil to lymphocyte ratio in obese and lean patients with polycystic ovary syndrome. J Endocrinol Invest 2016;39(1):4553 (doi:10.1007/s40618-015-0335-2).

34. Pinna A, Porcu T, D'Amico-Ricci G, Dore S, Boscia F, Paliogiannis $\mathrm{P}$, et al. Complete blood cell count-derived inflammation biomarkers in men with age-related macular degeneration. Ocul Immunol Inflamm 2019;27(6):932-6 (doi:10.1080/09273948.2018.1485960). 